\title{
Self-Mode-Locked Vertical-External-Cavity Surface-Emitting Laser
}

\author{
Arash Rahimi-Iman*ª ${ }^{\text {a }}$ Mahmoud Gaafar ${ }^{\mathrm{a}}$, Christoph Möller ${ }^{\mathrm{a}}$, Max Vaupel ${ }^{\mathrm{a}}$, Fan Zhang ${ }^{\mathrm{a}}$, \\ Dalia Al-Nakdali ${ }^{\mathrm{a}}$, Ksenia A. Fedorova ${ }^{\mathrm{b}}$, Wolfgang Stolz ${ }^{\mathrm{a}, \mathrm{c}}$, Edik U. Rafailov ${ }^{\mathrm{b}}$, and Martin Koch ${ }^{\mathrm{a}}$ \\ ${ }^{\mathrm{a} D e p a r t m e n t ~ o f ~ P h y s i c s ~ a n d ~ M a t e r i a l s ~ S c i e n c e s ~ C e n t e r, ~ P h i l i p p s-U n i v e r s i t a ̈ t ~ M a r b u r g, ~ R e n t h o f ~ 5, ~ D-~}$ \\ 35032 Marburg, Germany; \\ ${ }^{\mathrm{b}}$ Optoelectronics and Biomedical Photonics Group, School of Engineering and Applied Science, \\ Aston University, Aston Triangle, Birmingham B4 7ET, UK; \\ ${ }^{\mathrm{c}} \mathrm{NAsP}$ III/V GmbH, Am Knechtacker 19, 35041 Marburg, Germany; \\ *arash.rahimi-iman@physik.uni-marburg.de
}

\begin{abstract}
Ultrashort laser pulses from vertical-external-cavity surface-emitting lasers (VECSELs) have been receiving much attention in the semiconductor laser community since the first demonstration of sub-ps-pulsed devices more than a decade ago. Originally relying on semiconductor saturable-absorber mirrors for pulse formation, mode-locked operation has not only become accessible by using a variety of saturable absorbers, but also by using a saturable-absorber-free technique referred to as self-mode-locking (SML). Here, we highlight achievements in the field of SML-VECSELs with quantum-well and quantum-dot gain chips, and study the influence of a few VECSEL parameters on the assumed nonlinear lensing behavior in the system.
\end{abstract}

Keywords: Semiconductor disk lasers, vertical-external-cavity surface-emitting laser, self-mode-locking, mode-locked lasers.

\section{INTRODUCTION}

Within the last 15 years, a new class of pulsed lasers emerged which proved capable of generating ultrashort pulses. Optically pumped semiconductor lasers (OPSLs), also referred to as semiconductor disk lasers (SDLs), or verticalexternal-cavity surface-emitting lasers (VECSELs), have become promising sources of fs-pulsed laser light which indeed can serve many applications, such as ultrafast spectroscopy, metrology, multiphoton microscopy, or material processing. Moreover, the external-cavity design of such lasers allows for versatility, compactness and beam quality aspects which are all beneficial to the employment of VECSELs. While this type of device has a history of nearly fifty years following the proposal by Basov et al. in 1966 [1], the first of its kind under optical pumping was demonstrated by Kuznetsov et al in 1997 [2], giving rise to a new class of lasers. Shortly after, the first mode-locked VECSEL was presented to the community by Hoogland et al [3], which however slowly triggered efforts at the beginning to improve the device features. Nevertheless, after 2005, a significant increase of publications was registered, as the chart in Fig. 1 shows (distinguishing between saturable-absorber-based and saturable-absorber-free devices, and theoretical considerations, respectively; counts given to the best of our knowledge).

Much work has been performed in the field of saturable-absorber-based pulsed VECSELs by the community to provide ever shorter pulses using resonator-integrated [4-6] or even chip-integrated [7] semiconductor saturable-absorber mirrors (SESAMs), which have demonstrated an excellent performance of passively mode-locked VECSELs with pulse durations in the range of 100 fs [6] to picoseconds [3]. With new chip designs proposed and studied [8], which optimize gain dynamics and group-delay dispersion, ultrashort pulse formation is at the horizon which could eventually undercut the $100 \mathrm{fs}$ margin for pulse durations with unambiguous results. Up-to-date, the improvement of several aspects such as the average output power, peak power, pulse duration and repetition rate have been individually pushed forward, the results of which are summarized in Table 1. Although VECSEL operation is not limited to the InGaAs-on-GaAs material system, it is worth to note that the listed record values were all obtained from InGaAs quantum wells as gain medium. This is related to the performance of GaAs based devices and the material properties, which also allowed for record-high

Vertical External Cavity Surface Emitting Lasers (VECSELs) VI, edited by Keith G. Wilcox, Proc. of SPIE Vol. 9734, 97340M · C 2016 SPIE · CCC code: 0277-786X/16/\$18 · doi: 10.1117/12.2216469 
continuous wave (CW) output exceeding 100 Watts from one chip [9]. Remarkably, mode-locked VECSELs can reach average powers as high as $5.1 \mathrm{~W}$ [10], and peak powers as high as $4.35 \mathrm{~kW}$ [5] have been demonstrated with SESAMbased devices, respectively.

Table 1. Overview of record results achieved with SESAM mode-locked QW-SDLs.

\begin{tabular}{|c|c|c|c|c|c|}
\hline & Record value & \#QWs & $\begin{array}{l}\text { Wavelength } \\
\text { (nm) }\end{array}$ & Year & Ref. \\
\hline Peak power & $4.35 \mathrm{~kW}$ & 10 & 1013 & 2013 & {$[5]$} \\
\hline Pulse duration & $107 \mathrm{fs}$ & 4 & 1030 & 2011 & [6] \\
\hline Average power & $5.1 \mathrm{~W}$ & 10 & 1030 & 2012 & [10] \\
\hline \multirow[t]{2}{*}{ Repetition Rate } & Highest: $50 \mathrm{GHz}$ & 7 & 958 & 2006 & {$[11]$} \\
\hline & Lowest: $85.7 \mathrm{MHz}$ & 16 & 989 & 2013 & [12] \\
\hline
\end{tabular}

However, in addition to the aforementioned advantages of mode-locked VECSELs, with respect to a practical use, also robustness, flexibility and cost-efficiency play a role, which can be addressed via an approach referred to as saturableabsorber-free mode-locking, or self-mode-locking (SML). SML VECSELs can circumvent limitations naturally imposed on the device's performance by SESAMs, which are costly additional intra-cavity elements prohibiting employment of a compact linear cavity configuration and have to be individually designed for a certain operation wavelength. Moreover, due to their absorbance, additional losses and heat dissipation have to be coped with.

In the following, we will give an overview on SML VECSELs [13-19], from which record-high peak-powers up to 6.8 kW [14] and sub-500-fs pulses [15] were obtained individually, and highlight recent activities in this field involving both quantum-well [16,19] as well as quantum-dot based [17] gain media. Furthermore, assuming a nonlinear lensing effect to play a significant role for SML operation [14-17], we have studied the influence of a few VECSEL parameters on SML, such as power densities and cavity geometries, in the context of recent considerations of a nonlinear refractive index [20]. However, further studies are necessary to elaborate on the mechanism behind self-mode-locking as it has not been understood, yet.

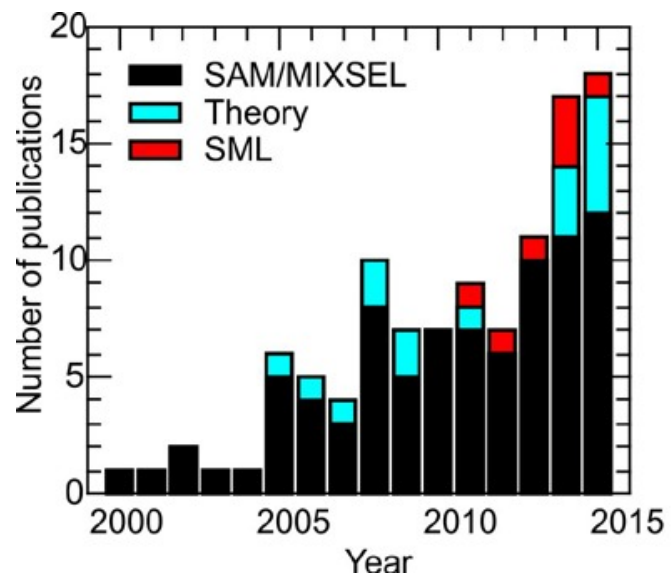

Fig. 1. Number of publications in the field of mode-locked VECSELs. SAM/MIXSEL represents saturable-absorberbased results, Theory includes studies on the gain region and reviews, SML highlights self-mode-locking results.

\section{THE FIRST RESULTS}

SESAM-free mode-locking of VECSELs was first reported in 2011 by Chen et al., using a regular straight cavity configuration [13]. To explain the observed behavior, the authors suggested that mode-locking resulted from saturable absorption in the unpumped quantum wells of the gain region, analog to chip-integrated SESAM structures. At that time, pulse durations as short as $654 \mathrm{fs}$ were obtained with an average output power from the device of $0.45 \mathrm{~W}$ at a repetition 
rate $2.17 \mathrm{GHz}$. Almost simultaneously, Kornaszewski et al. published their version of an SML VECSEL using a folded six-mirror cavity corfiguration [14]. However, the origin of pulsed operation was attributed to an i ntensity dependent Kerr-lens effect taking place in the semiconductor gain medium. In that work, mode-locked operation was shown for two different configurations. Firstly, stable mode-locking was observed when the cavity operated near its stability limit. From that, single pulses with duration of $\sim 1.5$ ps were obtained, with the VECSEL emitting an average output power of $700 \mathrm{~mW}$ at $200 \mathrm{MHz}$ repetition rate. Secondly, mode-locking was also achieved with the cavity being operated in its stability region together with an intra-cavity hard aperture inserted in front of the output coupler. Thereby, pulses with duration of $930 \mathrm{fs}$ were achieved, at similar wavelength and repetition rate with an average output power of $1.5 \mathrm{~W}$, but a record-high $6.8 \mathrm{~kW}$ peak power [14].

In fact, in the initial stage, SML reports gave rise to some controversy, partially due to a lack of complete or unambiguous characterization of the emission regime, but also due to fact that the mechanism responsible for self-modelocking has not been clear. The most prominent discussion on the validity of self-mode-locking results is presented in Refs. [21,22], triggered by the second report on SESAM-free ML-VECSEL [14].

In Ref. [21], the authors state that additional experimental evidences are required to claim stable and true mode-locked operation, including long-time-span intensity autocorrelation and pulse train measurements, in order to confirm that no coherent artifact or CW component is present and that the laser runs in a stable mode-locked regime, respectively. Also reference to features in radio-frequency signal is given. Indeed, later publications have taken into account these remarks to bolster up the claim of an SML regime.

\section{SML DEVICES}

Table 2. Overview on SML VECSEL structures and reported results (records highlighted).

\begin{tabular}{|c|c|c|c|c|c|c|c|}
\hline Year & $\begin{array}{l}\text { Wavelength } \\
(\mathrm{nm})\end{array}$ & Gain Region & $\begin{array}{l}\text { Pulse } \\
\text { Duration } \\
\text { (fs) }\end{array}$ & $\begin{array}{l}\text { Peak Power } \\
\text { (W) }\end{array}$ & $\begin{array}{l}\text { Average Power } \\
\text { (W) }\end{array}$ & $\begin{array}{l}\text { Repetion } \\
\text { Rate (GHz) }\end{array}$ & Ref. \\
\hline \multirow[t]{3}{*}{2011} & 1064 & QW & 778 & - & 2.35 & 2.17 & 13 \\
\hline & & & 654 & - & 0.45 & & \\
\hline & & & 1170 & - & 5.1 & & \\
\hline 2012 & 985 & QW & 930 & 6800 & 1.5 & 0.21 & 14 \\
\hline \multirow[t]{4}{*}{2013} & 1026 & QW & 1320 & - & - & 1 & 15 \\
\hline & & & 1010 & - & - & 1 & \\
\hline & & & 758 & - & - & 1 & \\
\hline & & & 482 & - & - & 1 & \\
\hline \multirow[t]{4}{*}{2014} & 1038 & QD & $>830$ & $<460$ & $<0,75$ & 1.5 & 17 \\
\hline & 1014 & QW & 860 & 948 & - & 0.504 & 16 \\
\hline & & & 1120 & 752 & - & 1.008 & \\
\hline & & & 950 & 754 & - & 1.512 & \\
\hline 2015 & 1059 & QW & 2350 & - & - & 1.567 & 23 \\
\hline
\end{tabular}


In the following period, also other groups demonstrated self-mode-locking: Albrecht et al. investigated a cavity using both soft and hard apertures to mode-lock their device, assuming that a negative Kerr-lensing effect is involved [14], using a V-cavity design with an intra-cavity aperture. In 2014, even harmonic self-mode-locking was reported by Gaafar et al up to the third harmonic [16], with a z-shaped resonator design and an intra-cavity slit. When designing the cavity configuration, the authors assumed the chip to act as a negative Kerr-medium. For this VECSEL, not only a reasonably long pulse train and clear radio-frequency spectra were presented [16], but also a long-time-span autocorrelation trace, beam-profile measurement and extra-cavity second-harmonic generation, respectively [19] Additionally, self-modelocking was demonstrated for the first time with a quantum-dot chip in a regular straight cavity [17], wherein positive Kerr-lensing in the chip was assumed. Here, a brief summary on SML VECSEL structures and achieved results will be given in Table 2. Details on the used structures and resonator designs can be found in the literature.

Next, the quantum-well chip, which has been used by us for the study of self-mode-locking, is briefly described. Here, the design is common to CW devices. 10 (InGa)As quantum wells (QWs) equally spaced by $\lambda / 2(\mathrm{GaP})$ As barrier layers were incorporated into an MOVPE-grown VECSEL chip. The integrated distributed Bragg reflector (DBR) consists of $24 \frac{1}{2}$ pairs of $\lambda / 4 \mathrm{GaAs} /(\mathrm{AlGa})$ As layers. The whole structure other than the spacers in the active region is transparent to the wavelength of the fiber-coupled $808 \mathrm{~nm}$ diode pump laser. The "bottom-up" grown structure was later flip-chip bonded with Au-In solid-liquid interdiffusion onto a $350 \mu \mathrm{m}$-thick CVD diamond heat spreader. To promote modelocked operation, the cap layer thickness was reduced from $\lambda / 2$ (corresponding to the design for a resonant chip at 1010 $\mathrm{nm})$ to $\lambda / 4$ via wet etching in order to obtain an anti-resonant micro-cavity with minimized group-delay dispersion (GDD) and a spectrally broadened effective gain of the structure [16,19].

\section{POWER-DEPENDENT RESULTS FOR A LINEAR CAVITY}

(a)

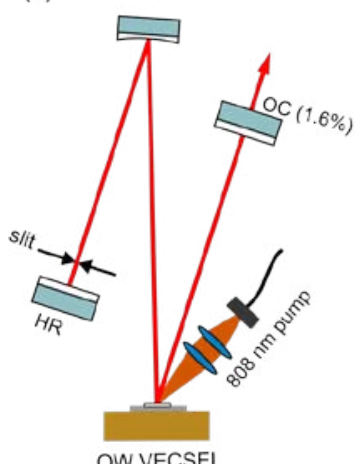

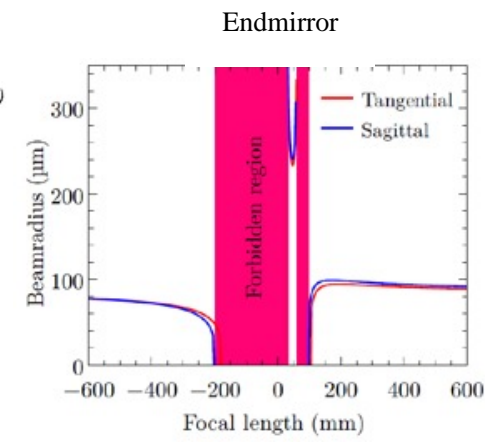

(b)

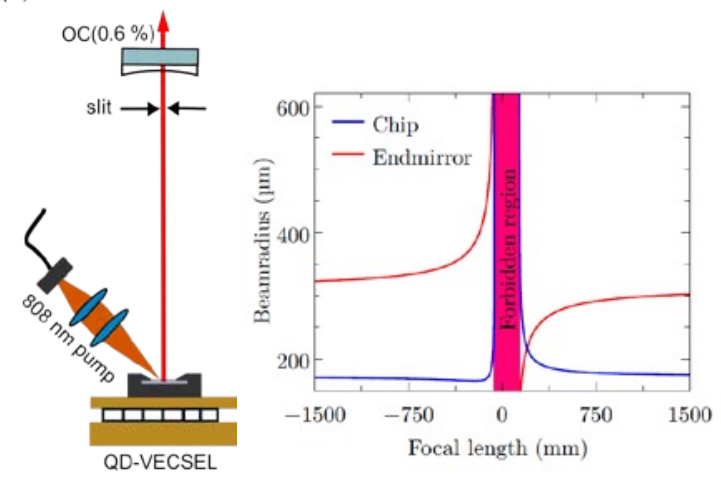

Fig. 2. a) Z-cavity configuration and b) linear-cavity VECSEL configuration for self-mode-locking, respectively, together with the corresponding evolution of the intra-cavity beam radius as a function of a Kerr-lens' focus length.

Considering the intensity-dependent Kerr-lensing effect as the basic cause for self-mode-locking, we used a simple model to calculate the beam radius inside the Z- and linear cavity, respectively, as a function of a focal length of a supposed lens. Using ABCD-matrix calculations for the beam radius, we added a thin lens at the VECSEL-Chip position to the cavity elements. The assumed Kerr-lens' focal length can be described by $f_{K r r r}=\frac{\pi \omega^{4}}{n_{2} L P}$ with beam radius $\omega$ at the lens position, thickness of the Kerr-medium $L$ and the power of the Laser beam $P . n_{2}$ is the nonlinear refraction index, which is the only variable in this equation that can be negative. Therefore the algebraic sign of the focal length is directly connected to the nonlinear refraction index. Supported by experimental data, Quarterman et.al [20] indicated that the nonlinear refraction index of a QW-VECSEL chip changes with increasing pump intensity from negative to positive values, with magnitudes amounting to $10^{-12} \mathrm{~cm}^{2} / \mathrm{W}$ using probe pulses in the picosecond region. Taking this behavior into account, we calculated the beam radius at the end mirror and at the chip for different focal length as shown in Fig 2. For a linear cavity, the beam radius increases for negative $n_{2}$ and high powers at the end mirror. Therefore, a hardaperture mode-locking is not possible for a negative nonlinear refraction index. A positive $n_{2}$ instead leads to a tapering of the beam radius at the end mirror, which can be used to trigger mode-locking by introducing high losses for the CW laser mode with a slightly closed slit. The same assumptions transferred to the Z-cavity used in the work of Gaafar et.al. 
[16] show a different behavior. The beam radius at the end mirror, as shown in Fig 2, experiences a tapering for both, positive and negative nonlinear refraction index.

Experiments on power-dependence:
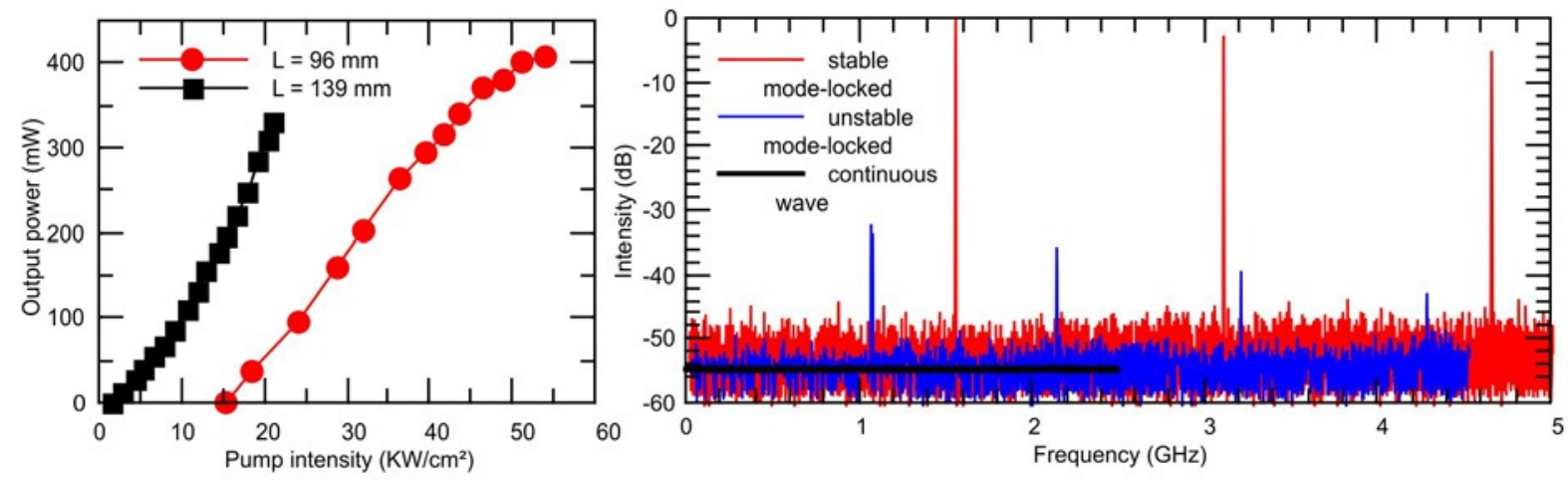

Fig. 3 a) Power curve of two linear cavities of different length. b) RF spectra showing three regimes of operation as a function of the optical power densities: continuous-wave, unstable and stable mode-locked operation, respectively.

The linear cavity allows us to test the power-dependence of the assumed nonlinearity for a VECSEL in action without undertaking a Z-scan experiment. To demonstrate the impact of optical intensities on the self-mode-locking behavior, we used two different linear cavities for this experiment. The first cavity with a length of 139 mm consisted of a highreflective end mirror with $200 \mathrm{~mm}$ radius of curvature, while the second cavity employs a 1\%-transmission output coupler with $100 \mathrm{~mm}$ radius of curvature. As apparent in the power curve in Fig 3a, we used these two cavities to enter different pump-density regimes.

At an optical pump density of $6.5 \mathrm{KW} / \mathrm{cm}^{2}$, we could only obtain a stable CW operation. Closing the slit in front of the end mirror only reduced the output power, but no sights of pulsing were apparent. The following increase of the pump density to $13.8 \mathrm{KW} / \mathrm{cm}^{2}$ allowed for a semi-stable pulsed operation. An offset was obtained in the measured pulse train and the shown RF-spectrum features a signal-to noise ration of $25 \mathrm{dBc}$ for the fundamental mode, which is not sufficient for stable mode-locking. Furthermore the harmonics drop in intensity very fast, which is also a sign of instabilities in this state. The corresponding RF-spectra of this experiment at different pump-power levels are shown in Fig. 3b.

At a pump density of $41.8 \mathrm{KW} / \mathrm{cm}^{2}$, we achieved stable self-mode-locked operation, comparable to power-dependent observations made in Ref. [14]. Clean pulses with little disturbances, which can be explained by the too small bandwidth of photodiode and oscilloscope for this high repetition rate, were obtained in a pulse train measurement. The RF spectrum shows stable pulsing with a narrow fundamental mode at $1.56 \mathrm{GHz}$ with a FWHM of $70 \mathrm{kHz}$. The achieved output power of $315 \mathrm{~mW}$ together with the measured pulse duration of 3.5ps leads to a pulse peak-power of around 55 $\mathrm{W}$, which corresponds to an intra-cavity peak-power of $5.5 \mathrm{KW}$. Using the given equation for the Kerr-lens with $P \sim 5.5$ $\mathrm{KW}, L \sim 5 \mu \mathrm{m}$ and $\omega$ as a function of the focal length of the assumed lens, one can derive a nonlinear refractive index of $10^{-16} \mathrm{~cm}^{2} / \mathrm{W}$ to $10^{-18} \mathrm{~cm}^{2} / \mathrm{W}$ which are in agreement with the measurement results of Quarterman et.al. [20].

These results hint at the possible fact that at pump intensities $I_{\text {pump }}$ around $13 \mathrm{KW} / \mathrm{cm}^{2}$ the sign of $n_{2}$ changes from negative to positive for this specific VECSEL chip. Considering these results, we can derive an explanation for the stable regimes of harmonic self-mode-locking described by Gaafar et.al. [16]. In that z-cavity device, three stable regions were found at $I_{\text {pump }}=4.7 \mathrm{KW} / \mathrm{cm}^{2}, 6.7 \mathrm{KW} / \mathrm{cm}^{2}$ and $7.7 \mathrm{KW} / \mathrm{cm}^{2}$ with one, two and three pulses in the cavity, respectively. With only minor differences given between the z- and l-cavity configuration in terms of pumping and lasing regime, one can assume an approximately equal behavior of $n_{2}$. Therefore all three plateaus at given pump densities are presumably in a region of a negative nonlinear refraction index. All regions of stable self-mode-locking are characterized by a comparable pulse duration and peak-power. In contrast, a change in the pulse power at a fixed $n_{2}$ would lead to a different focal length and therefore to instabilities, which was observed by Gaafar et al. in-between stable regimes, which are equally separated on the power scale. Thus, at certain pump levels, the average power is eventually high enough to lead to a circulation of an additional pulse in the resonator, whereby the optical intensity is split. With the higher-harmonic pulses exhibiting nearly the same properties of the fundamental regime, i.e. pulse duration and peak power, the focal length of the intensity-dependent Kerr-lens effect may be preserved at these pump-power levels. 


\section{CONCLUSIONS}

Recent advances in the field of self-mode-locked VECSELs, which are promising sources of pulsed light based on compact semiconductor disk lasers, have been summarized in the context of general developments regarding modelocked operation. Various examples of SML-VECSELs have been demonstrated, with peak powers up to $6.8 \mathrm{~kW}$, or pulse durations as short as hundreds of fs. Moreover, results obtained for both quantum-well and quantum-dot gain chips at repetition rates between $200 \mathrm{MHz}$ and $2 \mathrm{GHz}$ show that flexibility in the VECSEL design is given. Moreover, owing to the saturable-absorber-free design, features such as cost-efficiency, wavelength-versatility and better performance become appealing for applications. With the mechanism behind SML still unexplored, additional studies will be necessary to identify nonlinear effects in the chip, which are assumed to be responsible for an effective pulse formation.

\section{ACKNOWLEDGEMENTS}

The authors acknowledge financial support by the DFG (GRK 1782 and SFB 1083) and EU FP7 program through FAST-DOT project (contract No. 224338). M. Gaafar acknowledges support from the Yousef Jameel scholarship funds.

\section{REFERENCES}

[1] Basov, N. G., Bogdankevich, O. V., and Grasyuk, A. Z., IEEE J. of Quantum Electron. 2, 594-597 (1966).

[2] Kuznetsov, M., Hakimi, F., Sprague, R., and Mooradian, A., IEEE Photonics Technol. Lett. 9, 1063-1065 (1997).

[3] Hoogland, S., Dhanjal, S., Tropper, A. C., Roberts, S. J., Häring, R., Paschotta, R., and Keller, U., IEEE Photon. Technol. Lett. 12, 1135-1137 (2000).

[4] Keller, U., and Tropper, A. C., Physics Reports 429, 67-120 (2006).

[5] Wilcox, K.G., Tropper, A.C., Beere, H.E., Ritchie, D.A., Kunert, B., Heinen, B., Stolz, W., Opt. Express 21(2), 1599-1605 (2013)

[6] Klopp, P., Griebner, U., Zorn, M., and Weyers, M., Appl. Phys. Lett. 98, 071103 (2011).

[7] Mangold, M., Zaugg, C. A., Link, S. M., Golling, M., Tilma, B. W., and Keller, U., Opt. Express 22, 6099-6107 (2014).

[8] Kilen, I., Hader, J., Moloney, J. V., and Koch, S. W., Optica 1, 192-197 (2014)

[9] Heinen, B., Wang, T. L., Sparenberg, M., Weber, A., Kunert, B., Hader, J., Koch, S. W., Moloney, J. V., Koch, M., and Stolz, W., Electron. Lett. 48, 516-517 (2012).

[10] Scheller, M., Wang, T. L., Kunert, B., Stolz, W., Koch, S. W., and Moloney, J. V., Electron. Lett. 48, 588-589 (2012).

[11] Lorenser, D., Maas, D. J. H. C., Unold, H. J., Bellancourt, A. R., Rudin, B., Gini, E., Ebling, D., and Keller, U., IEEE J. Quantum Electron. 42, 838-847 (2006).

[12] Butkus, M., Viktorov, E. A., Erneux, T., Hamilton, C. J., Maker, G., Malcolm, G. P. A., and Rafailov, E. U., Opt. Express 21, 25526-25531 (2013).

[13] Chen, Y. F., Lee, Y. C., Liang, H. C., Lin, K. Y., Su, K. W., and Huang, K. F., Opt. Lett. 36, 4581-4583 (2011).

[14] Kornaszewski, L., Maker, G., Malcolm, G. P. A., Butkus, M., Rafailov, E. U., and Hamilton, C. J., Laser Photonics Rev. 6, L20-L23 (2012).

[15] Albrecht, A. R., Wang, Y., Ghasemkhani, M., Seletskiy, D. V., Cederberg, J. G., and Sheik-Bahae, M., Opt. Express 21, 28801-28808 (2013).

[16] Gaafar, M., Möller, C., Wichmann, M., Heinen, B., Kunert, B., Rahimi-Iman, A., Stolz, W., and Koch, M., Electron. Lett. 50, 542-543 (2014).

[17] Gaafar, M., Al Nakdali, D., Möller, C., Fedorova, K. A., Wichmann, M., Shakfa, M. K., Zhang, F., Rahimi-Iman, A., Rafailov, E. U., and Koch, M., Opt. Lett. 39, 4623-4626 (2014).

[18] Liang, H. C., Tsou, C. H., Lee, Y. C., Huang, K. F., and Chen, Y. F., Laser Phys. Lett. 11, 105803 (2014).

[19] Gaafar, M., Richter, P., Keskin, H., Möller, C., Wichmann, M., Stolz, W., Rahimi-Iman, A., and Koch, M., Opt. Express 22, 28390-28399 (2014).

[20] Quarterman, A. H., Tyrk, M. A., and Wilcox, K. G., Appl. Phys. Lett., 106, 011105 (2015).

[21] Wilcox, K. G., and Tropper, A. C., Laser Photonics Rev. 7, 422-423 (2013).

[22] Kornaszewski, L., Maker, G., Malcolm, G. P. A., Butkus, M., Rafailov, E. U., and Hamilton, C. J., Laser Photonics Rev. 7, 555-556 (2013).

[23] Tsou, C. H., Liang, H. C., Wen, C. P., Su, K. W., Huang, K. F., and Chen, Y. F., Opt. Express 23, 16339-16347 (2015) 